\title{
Cherubism, a rare childhood disease with a brief review of literature
}

\author{
Dr. Kaushal Shah ${ }^{1}$, Dr. Amol Karagir ${ }^{2}$, Dr. Rajesh Koppikar ${ }^{3}$, \\ Dr. Sampada Kanitkar ${ }^{4}$ \\ ${ }^{1,2}$ (Department of Oral Medicine \& Radiology, Bharati Vidyapeeth Deemed University Dental College, Sangli, \\ Maharashtra, India.) \\ ${ }^{3}$ (Department of Periodontology, Bharati Vidyapeeth Deemed University Dental College, Sangli, Maharashtra, \\ India) \\ ${ }^{4}$ (Department of Oral Pathology, Bharati Vidyapeeth Deemed University Dental College, Sangli, Maharashtra, \\ India)
}

\begin{abstract}
Cherubism is a rare, benign, non-neoplastic fibro-osseous hereditary condition related to genetic mutations, characterised by symmetrical expansion of the mandible and/or maxilla, usually found in children between 2-5 years of age giving characteristic cherubic appearance and an upward turning of the eyes. These lesions gradually increase for next few years, stay inactive and start regressing after puberty. On radiography, the lesions exhibit bilateral multilocular radiolucent areas. Histopathology reveals multinucleated giant cells in the background of proliferating fibrous connective tissue. These self-limiting, spontaneously regressing lesions do not require any surgical intervention, except for significant functional and/or esthetic impairment. The present case describes Cherubism in a young child who is far from reaching puberty, with a brief review of literature on cherubism.
\end{abstract}

Keywords: cherubism, childhood disease, fibro-osseous lesion, hereditary, self-limiting.

\section{Introduction}

Cherubism is a rare, autosomal dominant, non-neoplastic fibro-osseous hereditary condition related to genetic mutations, characterised by symmetrical expansion of the mandible and/or maxilla, usually found in children between 2-5 years of age giving characteristic cherubic appearance and an upward turning of the eyes. ${ }^{1}$ These lesions gradually increase for next few years, stay inactive and undergo spontaneous regression after puberty. Although the histopathologic characteristics of cherubism are not pathognomic, the diagnosis is strongly suggested by bilateral, relatively symmetric jaw involvement that is limited to maxilla and mandible.

And imaging which typically shows expansile remodeling of involved bones, thining of cortexes and multilocular radiolucencies with coarse trabecular pattern. ${ }^{2}$

Cherubism should be considered in the differential diagnosis of children and young patients presenting with bilateral mandibular swelling, as they can mimic gigantiform cementoma, odontogenic myxoma, giant cell granuloma of the mandible or pathologies related to parotid glands. ${ }^{3} \mathrm{~A}$ comprehensive evaluation of the family history, clinical, and radiological features can help confirm the diagnosis. Once diagnosed as Cherubism, depending on the severity or grade, no specific treatment may be necessary, as compared to other pathologies, since these expansile lesions have a tendency to spontaneously regress after puberty. ${ }^{3}$ At most, a periodic follow-up is all that is necessary to deal with any residual esthetic or functional impairment.

\section{Case Report}

We present a case of a young child who was brought by the parents to our out-patient department with a concern for the slowly growing bilateral swellings of the cheeks. According to the parents, the child was normal at birth, however they noticed painless bilateral jaw enlargements around 3 years of age, which gradually increased till the child was about 7 years of age. However there has been little change since then, though they have noticed increased crowding of teeth. There was no history of any prodromal symptoms or trauma nor any difficulty in speaking, chewing, or swallowing. There was no relevant family history either.

Patient was conscious, co-operative and well oriented in time, space and person, having normal gait, erect posture and average built.

\subsection{Clinical Examination}

On inspection the lower third of the face appeared to be bilaterally expanded giving a chubby cheek appearance (Fig.1) No change in color and texture of overlying skin was noted. On palpation, the swellings were hard, firm and non tender, with no increase in local temperature. The eyes appeared to be upturned revealing the 
white sclera below (Fig.2), while the remaining head, face and neck structures appeared normal. The temporomandibular joint and parotid salivary glands too showed no abnormality. However, intra-oral examination revealed a mixed dentition, with many over-retained deciduous teeth $(52,53,54,55,61,62,63,73,74,75,83,84,85)$ and delayed eruption of the permanent teeth, for the age. The intraoral soft tissues appeared normal.

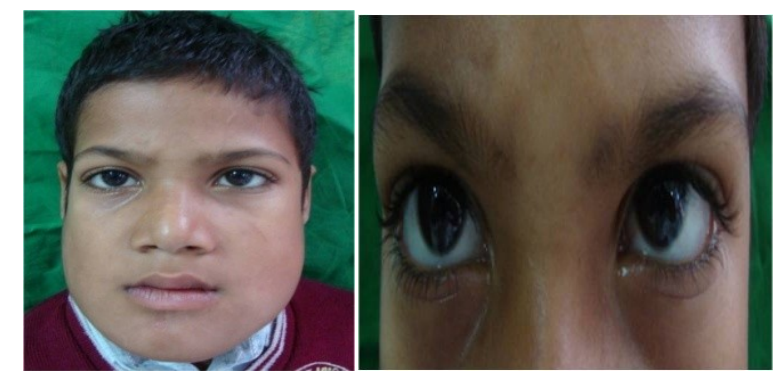

Fig.1Front Facial Photograph Fig.2 close-up of the eyes

\subsection{Radiographic Examinations}

Various conventional views and specialised view were taken.

\subsubsection{Orthopantomograph}

(OPG) revealed multiple developing permanent teeth $(12,13,14,15,17,22,23,27,33,34,35,37,43,44,45,47)$ and over-retained deciduous teeth $(52,53,54,55,61,62,63,73,74,75,83,84$,and 85$)$. Bilateral osteolytic multiloculated lesions were seen involving the entire body and vertical and horizontal ramus of the mandible, blunting the coronoid processes, but sparing the condyles. There was no sign of maxillary involvement. (Fig.3)

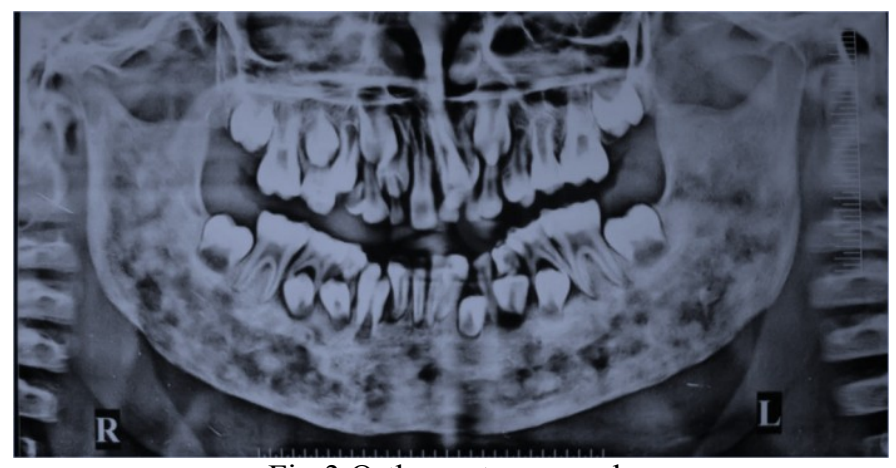

Fig.3 Orthopantomograph

\subsubsection{Postero-anterior}

(PA) skull showed extensive multilocular radiolucent expansile lesions involving the body and ramus of mandible bilaterally, causing expansion and thinning of the buccal and lingual cortices but no periosteal reaction. Extension upto the coronoid processes was evident causing its blunting. No involvement of maxillary bone or orbits evident. A thickened soft-tissue shadow around the ramus was also perceived, giving a visibly chubby clinical appearance. (Fig 4)

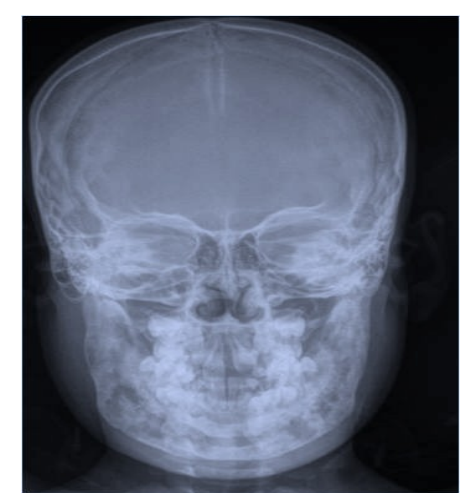

Fig. 4 Postero-anterior Skull View 
2.2.3 Lateral oblique radiographs of both right and left mandibles also showed similar multilocular radiolucent lesions causing expansion of the entire mandible, except the condyles, and blunting of coronoids.(Fig.5)

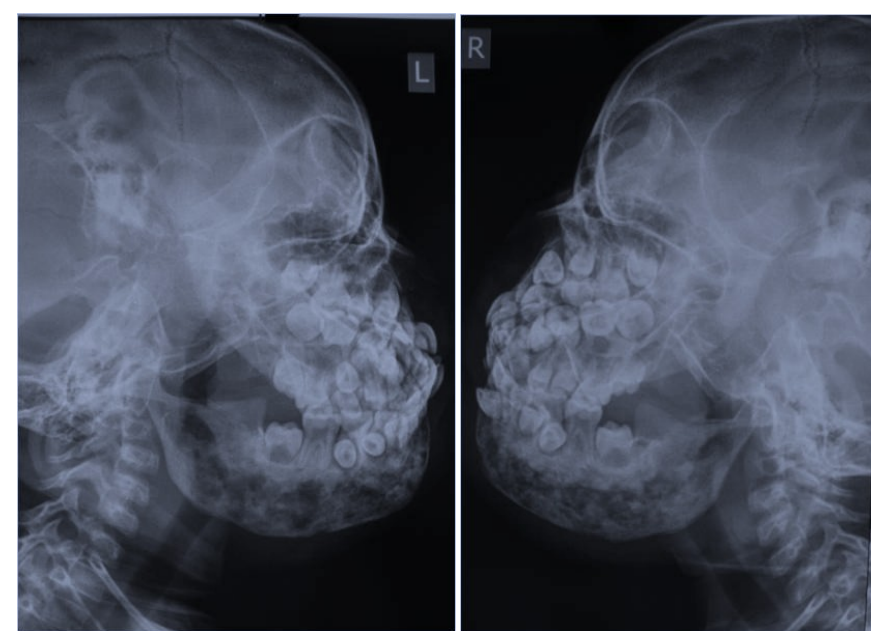

Fig.5 Left and Right Lateral Oblique Views

2.2.4 Computed tomographic (C. T.) Scans in coronal plane revealed a bilateral, abnormal radiolucent lesions with ground glass density causing expansion of the mandible involving the horizontal and vertical rami and the alveolar cortex. Non-involvement of condyle was evident (Fig.6), while an Axial CT revealed bucco-lingual expansion of thinned out mandibular cortex with multilocular radiolucencies and multiple over-retained deciduous teeth. (Fig.7)

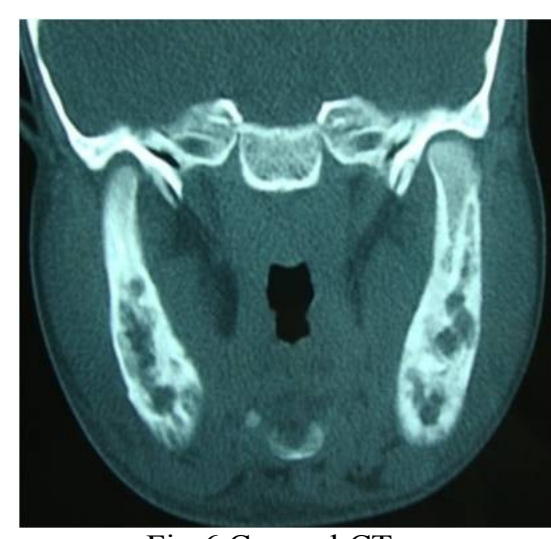

Fig.6 Coronal CT

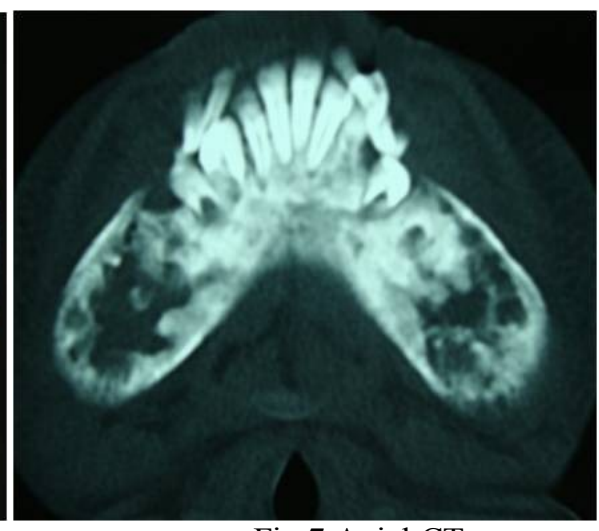

Fig.7 Axial CT

2.2.5 3-Dimensional computed tomographic (3D CT) reformatted images (Fig.8) too showed bilateral expansion of the body and rami of the mandible, along with crowding of teeth.

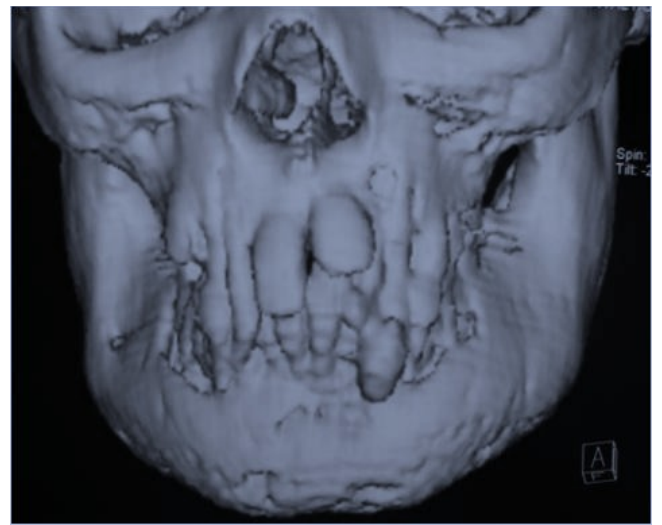

Fig.8 3D CT 


\subsection{Laboratory Investigations}

Routine blood investigation revealed low haemoglobin $(\mathrm{Hb})$ value of $8.1 \mathrm{gm} \%$, other values were within normal range. Serum calcium and serum alkaline phosphates were in normal range too. HIV antibody test was non reactive. While, T3, T4, and TSH levels were also normal.

\subsection{Histopathologic Examination}

Incisional biopsied, Haemotoxylin and Eosin (H\&E) stained section showed lesional tissue consisting of trabeculated bony tissue with osteocytes, and osteoblasts and some multinucleated giant cells. Connective tissue showed delicate fibrillar stroma with an increased number of blood vessels and capillaries. Suggestive of a fibro-osseous lesion.

Considering the aforementioned findings on radiology and histopathology, along with the help of clinical examination and history, a diagnosis of Cherubism was made.

\subsection{Outcome and Follow-up}

The outcome is favourable, as the disease undergoes spontaneous regression after puberty. Periodic long term follow-up is advised till puberty, to deal with any residual esthetic or functional problems, if they persist.

\section{Discussion}

Cherubism is a rare hereditary fibro-osseous childhood disease characterised by bone degradation and fibrous tissue replacement at the angles of the mandible and the

tuberosities of the maxilla that leads to prominence of the lower face. ${ }^{1,5}$ The disorder was first described in 1933 by Jones as a familial fibro-osseous disease involving the maxilla and the mandible. ${ }^{1,5} \mathrm{He}$ named it initially "Familial Multilocular Cystic Disease of the Jaws". Consequently, in 1938, Jones rechristened it as "Cherubism" to reflect characteristic chubby cherubic facial appearance of the children similar to those of Cherubs portrayed in Renaissance religious paintings. ${ }^{6}$

According to the World Health Organization classification, cherubism belongs to the non-neoplastic bone lesions group that involves the mandible. ${ }^{3,7}$ The disease can also be referred to as familial or hereditary fibrous dysplasia, bilateral giant cell tumour, or familial multiloculated disease. ${ }^{7,8}$ The pathogenesis of cherubism remains controversial. No cause and effect relationship with trauma, infection, or hemorrhage has ever been verified. Anderson suggested that a genetically induced biochemical abnormality stimulates the giant cell lesions characteristic of cherubism. A molecular pathogenesis of cherubism has been proposed; SH3BP2 gene mutations cause dysregulation of the Msx-1 gene, which is involved in regulating mesenchymal interaction in craniofacial morphogenesis. ${ }^{9}$

Affected children are normal at birth. Progression of the bone lesions usually slows down after the age of 5 years, and stops at the age of 12 to 15 years. When patients reach puberty, the osseous lesions of cherubism regress spontaneously. However, the underlying cause of this regression is not known. Mandibular lesions are usually painless and symmetrical. In general, cherubism does not affect other parts of the skeleton or osseous metabolism. ${ }^{5,10}$ Cherubism is reported to be associated with some well described syndromes, including neurofibromatosis type 1, Noonan-like/multiple giant cell lesion syndrome, Ramon syndrome, and JaffeCampanacci syndrome. ${ }^{11,12}$

Ramon and Engelberg ${ }^{13}$ have proposed a grading system for cherubism based on the area of involvement, as follows:

- grade 1 - involvement of both mandibular ascending rami

- grade 2 - grade 1 plus involvement of both maxillary tuberosities, and diffuse mandibular involvement

- grade 3 - massive involvement of the maxillae and mandibles except for the condylar processes

- grade 4 - grade 3 plus involvement of the floor of the orbits, causing orbital compression.

Our patient was somewhere between Grade 1 and Grade 2, as there was diffuse mandibular involvement, except for the condyles.

Histologically, the lesions are composed of a vascularized fibrous stroma containing multinucleated giant cells, resembling giant cell granuloma. ${ }^{7}$ Histopathologically, the lesions have been characterized into three subtypes: I-predominance of multinuclear cells; II-predominance of inflammatory activity; and IIIpredominance of fibrosis. Histopathology, by itself, sometimes is inconclusive to the diagnosis of the type of fibro-osseous lesion, as it was in our patient too.

Conventional radiography and $\mathrm{CT}$ scanning are more specific for the radiological diagnosis of cherubism. Bilateral multiloculated cystic expansions in the mandible is the characteristic radiological appearance. ${ }^{2,10}$ The most prominent radiological characteristics of the patients is the presence of expansile remodelling in the involved bones and distinctive cortical thinning. There is no accompanying periosteal reaction in the lesions. Mandibular involvement is typically bilateral and maxillary involvement always 
accompanies mandibular involvement. Lesions often begin near the angle of the mandible and expand into the body and ramus of the mandible. Expanding lesions often cause thinning of the cortex and, in the maxilla, may cause obliteration of the maxillary sinus. CT investigation is a better method for demonstrating expansile lesions and the soft tissue components in these lesions. ${ }^{14}$

For our patient, the OPG, PA Skull, lateral obliques and CT scans helped to provide a clear delineation of the extent of disease. They depicted the bilateral osseous involvement, cortical thinning, and expansile remodelling of involved bones. Also showed a multilocular appearance in the mandible created by the presence of bone septa and expansile remodelling and perforation in some places. Preservation of the mandibular condyles, was noted in our case too, like reported in many literature on cherubism. ${ }^{14}$ Additionally, 3D reformatting contributes to the diagnosis and surgical planning.

These conventional radiographic and CT findings of our patient were in concordant with reports in the literature $^{8,14,15}$ and helped reach diagnosis of Cherubism.

As the patient grows, the jaw bone tends to resolve and progressively resulting in a normal jaw configuration. ${ }^{16}$ Treatment of cherubism is not standardized. Surgical treatment appears to be unnecessary for Grade 1 and Grade 2 cases, in the absence of secondary disturbances. Liposuction has been proposed to reduce the mass of the lesion in particular cases. ${ }^{4}$ Curettage alone or in combination with surgical contouring for cosmetic purposes has been considered the treatment of choice for Grade 3 and Grade 4 cases. ${ }^{17}$ Some authors point to medical therapy in the form of calcitonin as a possibility to curtail the disease and obviate the need for surgery, but only a few references are available in the literature. ${ }^{18}$

In our case, the patient was categorized between Grade 1 and Grade 2, and was a long way before reaching puberty, and therefore only kept under periodic follow-ups. Specific management, if required will be undertaken after patient attains puberty.

\section{Conclusion}

Our case highlights the importance of the awareness of this rare syndrome that should be considered in the differential diagnosis of children and young patients presenting with bilateral mandibular swelling, where comprehensive evaluation of the clinical, radiological, and histopathological features can confirm the diagnosis, and help decide on the treatment. Once the diagnosis is established, genetic counselling of all family members is recommended. Frequent follow-ups are necessary and treatment if any is deferred till after puberty, patients having excellent prognosis thereafter.

\section{References}

[1] Khalifa MC, Ibrahim RA. Cherubism. J Laryngol Otol. 1988;102:568-570.

[2] Beaman FD, Bancroft LW, Peterson JJ, Kransdorf MJ, Murphey MD, Menke DM. Imaging characteristics of cherubism. AJR Am J Roentgenol. 2004;182:1051-1054.

[3] Yamaguchi T, Dorfman HD, Eisig S. Cherubism: clinicopathologic features. Skelet Radiol. 1999;28:350-353.

[4] Carvalho Silva E, Carvalho Silva GC, Vieira TC. Cherubism: clinicoradiographic features, treatment and long-term follow-up of 8 cases. J Oral Maxillofac Surg. 2007;65:517-522.

[5] Gomes MF, de Souza Setubal Destro MF, de Freitas Banzi EC, et al. Aggressive behaviour of cherubism in a teenager: 4-years of clinical follow-up associated with radiographic and histological features. Dentomaxillofac Radiol. 2005;34:313-318.

[6] Atalar MH, Albayrak E, Erdinc P, et al. Cherubism as a rare cause of bilateral expansion of the mandible: radiological manifestations. J HK Coll Radiol 2008;11:76-80.

[7] Regezi JA, Sciubba J. Oral pathology: clinical-pathologic correlations. 2nd ed. Philadelphia: Saunder;1993.p345-354

[8] Ozkan Y, Varol A, Turker N, et al. Clinical and radiological evaluation of cherubism: a sporadic case report and review of the literature. Int J Pediatr Otorhinolaryngol. 2003;67:1005-1012.

[9] Lo B, Faiyaz-Ul-Haque M, Kennedy S, et al. Novel mutation in the gene encoding c-Abl-binding protein SH3BP2 causes cherubism. Am J Med Genet A 2003;121A:37-40

[10] Ongole R, Pillai RS, Pai KM. Cherubism in siblings: a case report. J Can Dent Assoc. 2003;69:150-154

[11] Lakhkar BN, Lakhkar BB, Ghosh MK, et al. Cherubism. Indian Pediatr. 1990;27:1305-1307.

[12] Cohen MM Jr, Gorlin RJ. Noonan-like/multiple giant cell lesion syndrome. Am J Med Genet. 1991;40:159-166.

[13] Ramon Y, Engelberg IS. An unusually extensive case of cherubism. J Oral Maxillofac Surg. 1986;4:325-328.

[14] Beaman FD, Bancroft LW, Peterson JJ, et al. Imaging characteristics of cherubism. AJR Am J Roentgenol. 2004;182:1051-1054.

[15] Jain V, Sharma R. Radiographic, CT and MRI features of cherubism. Pediatr Radiol. 2006;36:1099-1104.

[16] Penarrocha M, Bonet J, Minguez JM, et al. Cherubism-a clinical, radiographic and histopathologic comparison of 7 cases. J Oral Maxillofac Surg 2006;64:924-930.

[17] Dukart RC, Kolodny SC, Polte HW et al. Cherubism: Report of case. J Oral Surg 1974;32:782-785.

[18] Teixeira VP, Dedivitis RA, Guimarães AV, Benetton AA, França CM. Cherubism-case report and literature review. Rev De Clín Pesq Odontol 2004;1:23-25 